\title{
HORMONAS FEMENINAS Y CANCER DE MAMA: ESTADO DE LA POLEMICA Y EVIDENCIAS EN 2008. ¿QUE RESPONDER A LAS PACIENTES?
}

\author{
Dr. Sergio Majlis D.
}

Unidad de Endocrinología. Clínica Alemana de Santiago - Chile.

\section{FEMALE HORMONES AND BREAST CANCER: CONTROVERSIES AND EVIDENCES AT 2008. WHAT TO ANSWER TO OUR PATIENTS?}

\begin{abstract}
The use of Hormone Replacement Therapy (HRT) has evolved from a massive utilization to a restricted use under very precise medical indications, in the light of the deletereous effects its indiscriminate prescription could entail. It is not beneficial for secondary prevention, but rather implies additional cardiocerebrovascular risks, and therefore it must be preferentially employed during perimenopausic and non-late periods, in the smallest possible dose and only over short periods of time, restricting its prescription to women presenting with severe estrogen deprivation syndrome or to osteoporotic patients intolerant to calcium and bifosfonates therapy. Breast cancer risk - which is low- is related to a estrogen theraphy extended for more than seven years or associated with the use of progesterone for a period exceeding five years. Personal medical records of breast cancer imply absolute contraindication. Currently, there are no research work supporting the safe use of phytoestrogens as an altenative therapy. It is likely that in upcoming days transdermal estrogen administration associated with natural progesterone could allow an increased use of them, but for the time being there are no massive studies supporting their recommendation.
\end{abstract}

Key words: Breast cancer, Hormone replacement therapy, Phytoestrogens.

Resumen: El uso de terapia de reemplazo hormonal ha evolucionado desde su empleo masivo a un uso restringido bajo indicaciones precisas, a la luz de los efectos deletéreos que pudiera significar su indicación indiscriminada. No es de utilidad en la prevención

Majlis S. Hormonas femeninas y cáncer de mama: Estado de la polémica y evidencias en 2008, ¿Qué responder a las pacientes? Rev Chil Radiol 2008; 14: 113-121.

Correspondencia: Dr. Sergio Majlis D.

majlissergio@entelchile.net secundaria y más bien representa riesgos adicionales cardiovasculares y cerebro vasculares, por lo que se debe emplear de preferencia en el periodo perimenopáusico y no tardío, en la menor dosis posible y por el menor tiempo necesario, restringiendo su indicación a las mujeres con síntomas de deprivación estrogénica severa o a mujeres osteoporóticas que no toleran la terapia de calcio y bifosfonatos. El riesgo de cáncer de mama - que es bajo-está relacionado con una terapia estrogénica prolongada de 7 años o más o en asociación con el empleo de progesterona por más de 5 años. Antecedentes personales de cáncer de mama representan una contraindicación absoluta. No hay trabajos a la fecha que apoyen el empleo sin riesgo de fitoestrógenos como una terapia alternativa al uso de estrógenos. Es posible que en el futuro la administración transdérmica de los estrógenos, asociado a progesteronas naturales permitan su mayor utilización, pero aún no se dispone de estudios masivos que aprueben su recomendación.

Palabras clave: Cáncer de mama, Fitoestrógenos, Terapia de reemplazo hormonal.

\section{Introducción}

El tratamiento hormonal de reemplazo (THR) permite - a corto plazo- tratar los signos y síntomas de climaterio. Durante años, hasta el 2002, se consideró como un elemento favorable en la prevención de numerosas patologías desde la cardiovascular al Alzheimer y, porqué no hablar, de la prevención del envejecimiento. Actualmente para muchos su uso se considera deletéreo.

El riesgo eventual de cáncer de mama es hoy por hoy, el obstáculo principal en la utilización prolongada de la THR. En una encuesta de National Council on the Aging (Editorial Lancet 1997), el 61\% de las mujeres interrogadas afirmó tener más miedo de adquirir un cáncer que temer del riesgo de enfermedades cardiovasculares, pese a que estas últimas representan la primera causa de mortalidad en mujeres.

Los trabajos de Magnusson ${ }^{(1)}$, Schrairer ${ }^{(2)}$, Ross ${ }^{(3)}$, publicados en 1999, 2000 y el estudio de Women Health Initiative (WHI) en $2002^{(4)}$, fueron ampliamente 
mediatizados generando actitudes apasionadas, a veces sin fundamento, de médicos y pacientes frente al uso de THR.

Se estima que aproximadamente el $30 \%$ de las mujeres entre 50 y 74 años siguen usando $\mathrm{THR}^{(5)}$, por lo que es de suma importancia evaluar el balance riesgo/beneficio a nivel poblacional.

Numerosas preguntas permanecen aún sin respuesta. Sin embargo, la información a las pacientes es esencial. ¿Qué responder a sus dudas y a las sensacionalistas revelaciones de los medios de comunicación?

Con el objetivo de facilitar esta tarea, analizaremos las evidencias disponibles para que cada persona pueda contar con un juicio informado.

\section{Algunos datos epidemiológicos}

El cáncer mamario constituye el cáncer más frecuente de la mujer: $50 \%$ de los cánceres femeninos antes de los 50 años de edad y $20 \%$ después de los 75 años. Existe una gran variabilidad geográfica en su incidencia: más elevada en el occidente, especialmente en EE.UU.: 75-80 cánceres por 100.000 mujeres, frecuencia netamente menor en Asia: 16 cánceres por 100.000 mujeres en Japón(6). Por otro lado, la incidencia de cáncer mamario está en aumento desde 1950, especialmente entre las mujeres postmenopáusicas de EE.UU. y norte de Europa, hecho que podría ser explicado por la implementación de campañas de screening.

Se estima actualmente que una americana de 50 años de edad tiene $10 \%$ de probabilidad de desarrollar un cáncer mamario y $3 \%$ de muerte por esta $\operatorname{causa}^{(7)}$.

La edad constituye el factor de riesgo más importante no modificable de esta enfermedad, siendo su incidencia mucho más alta en mujeres post-menopáusicas.

\section{Efecto de hormonas sexuales en la mama}

Es un hecho conocido que las hormonas femeninas se relacionan con el cáncer de mama: hace ya más de 100 años se practicaba la ooforectomía para detener la evolución del cáncer en mujeres premenopáusicas ${ }^{(8)}$.

Se plantean las siguientes interrogantes respecto al estrógeno:

\section{¿Como ejerce el estrógeno un rol deletéreo sobre la mama?}

El rol oncogénico de los estrógenos está dado en parte por la alquilación de sus moléculas a nivel celular, la generación de radicales que pueden dañar el DNA, junto con la potencial genotoxicidad de éste y algunos de sus metabolitos ${ }^{(9)}$. Los metabolitos oxidativos de los estrógenos afectan la proliferación y apoptosis del tejido mamario, por lo que son potencialmente carcinogénicos.
Tiene además un efecto proliferativo directo a través de la inducción de proteínas y enzimas que comprometen la síntesis de ácidos nucleicos e indirecto, estimulando factores de crecimiento (TGF- $\alpha$, IGF-1 entre otros).

La formación de un tumor ocurre en una glándula hormono-dependiente por una excesiva estimulación hormonal|(10) o por alteraciones en áreas específicas de transcripción del genoma, que llevan a una progresión desde un crecimiento normal, hiperplasia y por último a una transformación neoplásica.

Estudios recientes confirman el rol del estrógeno en la modulación de la angiogénesis tumoral a través de la estimulación de factores de crecimiento endotelial (VEGF) ${ }^{(11)}$ e inducción de la síntesis de enzimas (catepsina $\mathrm{D}$, activador de plasminógeno), que podría favorecer la diseminación me tastásica.

\section{¿Como actúa el estrógeno sobre el receptor nuclear?}

El estrógeno sólo puede ejercer su acción en presencia de receptores nucleares específicos $\alpha$ (ER $\alpha)$ y $\beta$ (ER $\beta$ ), cuya concentración es variable en los diferentes tejidos y de mujer en mujer, dependiendo además de otros factores como edad y etnicidad (mayor en blancas) $^{(12)}$.

El receptor ER $\beta$ disminuye la sensibilidad del receptor ER $\alpha$ y por lo mismo actúa como un regulador fisiológico, disminuyendo el rol proliferador ejercido por el primero. Ambos tienen una identidad aminoacídica del $96 \%$ pero sólo un $53 \%$ de homología en su fracción ligando, lo cual explica la gran especificidad en su respuesta. Su proporcionalidad es importante: frente a una mayor expresión de ER $\alpha$. vs ER $\beta$, se han reportado tumores más agresivos, mientras que la mayor presencia de receptores $\beta$ estaría más relacionada con la inhibición de la proliferación celular, por lo que se asocia con tumores menos agresivos y con un mayor intervalo libre de enfermedad. Parece existir un mayor riesgo de cáncer de mama a mayor concentración de receptores ${ }^{(13)}$. El $84 \%$ de los cánceres mamarios expresa receptores estrogénicos ${ }^{(14)}$, siendo por ésto hormono-dependientes; este hecho favorece el uso de quimio-prevención con SERM-s y boqueadores de aromatasa.

\section{¿En qué forma el medio hormonal endógeno incide en el cáncer de mama?}

Existe una conocida mayor asociación de cáncer mamario a:

* $\quad$ Menarquia precoz (< 12 años): por cada 2 años de retraso de la menarquia, hay un $10 \%$ de reducción de riesgo ${ }^{(15)}$

* $\quad$ Primiparidad tardía (> 30 años): tener un primer hijo a los 36 años tiene un riesgo 1,6 veces mayor de cáncer de mama que tenerlo a los 26 años ${ }^{(16)}$.

* Nuliparidad: representa un riesgo relativo (RR) de $1,08^{(17)}$. 
* Menopausia tardía: aumento de $1.03 \%$ de RR por cada año de retraso ${ }^{(18)}$.

Estos factores son consistentes con el rol acumulativo que tiene la exposición prolongada a estrógenos, aparentemente proporcional con los ciclos menstruales espontáneos ${ }^{(19)}$. Al combinarlos entre sí, su valor predictivo se incrementa(20).

Existen otros factores de riesgo conocidos, como el nivel de estrógenos endógenos que posee la paciente, el antecedente familiar en primer grado de cáncer de mama, la presencia de mutaciones BRCA1 y BRCA2, p53, ATM, PTEN, la ausencia de lactancia, la obesidad en la post-menopausia, los elevados estrógenos endógenos y hormonas sexuales de origen suprarrenal|(21). El haber tenido cáncer de mama previamente, incrementa el riesgo en el tejido mamario restante y en la mama contralateral en un $0,5 \%$ anual en post-menopáusicas y $1 \%$ en la pre-menopausia ${ }^{(22)}$. Estudios recientes incorporan las progestinas de la THR, como un factor de riesgo adicional(23).

\section{¿Las hormonas sexuales endógenas tienen algún rol protector?}

Existen factores protectores del cáncer de mama, como:

- Edad joven al primer parto: su mayor protección se expresa posterior a los 10 años de éste ${ }^{(24)}$.

- Alta paridad y lactancia prolongada: disminuyen los ciclos ovulatorios y la exposición estrogénica ${ }^{(25)}$.

- Aumento de ciclos anovulatorios en la pre-menopausia (mujeres delgadas, deportistas, oligomenorreicas) se asocia a menor riesgo de cáncer de mama ${ }^{(26)}$.

- Niveles elevados de progesterona en la pre-menopausia ejerce un papel protector ${ }^{(27)}$.

\section{¿De dónde provienen los estrógenos en la mujer post-menopáusica?}

En la mujer pre-menopáusica, los ovarios son la fuente principal de estrógenos y sólo un mínimo porcentaje proviene de su conversión en órganos periféricos. Al llegar a la menopausia, los estrógenos provienen exclusivamente de la aromatización periférica (hígado, músculo y tejido graso) de andrógenos de origen ovárico y suprarrenal(28).

Estos estrógenos débiles (estrona) posteriormente son catabolizados por la $17 \beta$-hydroxyesteroide deshidrogenada a estradiol (estrógeno de mayor potencia). Esta enzima posee una actividad mayor en tumores de mama que en el tejido mamario normal y genera así un ambiente local más estrogenizado y favorable para el tropismo celular ${ }^{(29)}$. Variaciones en la producción local o en el catabolismo estrogénico, generan diferencias de exposición del tejido mamario a estrógenos y conducen a un mayor o menor riesgo.

\section{¿Que rol juega la obesidad en el cáncer de mama?}

Las mujeres obesas post-menopáusicas poseen una menor concentración de proteína transportadora de estrógeno (SHBG) y por ende un mayor porcentaje de estrógenos libres y un mayor riesgo relativo de cáncer de mama (RR: 2.00-2.58)(30). Mujeres con IMC >33 tienen un 27\% de incremento de riesgo cáncer de mama comparado con aquellas de IMC $<21$. Al bajar de peso en más de $10 \mathrm{Kg}$, se reduce en un $57 \%$ el riesgo de cáncer de mama.

En las pre-menopáusicas, la obesidad parece jugar un rol protector ${ }^{(21)}$, relacionado a más frecuentes ciclos anovulatorios.

Mujeres obesas post-menopáusicas expresan un aumento en la actividad aromatasa del tejido adiposo mamario ${ }^{(32)}$ la cual va a convertir los andrógenos de fuente suprarrenal y ovárica a mayores niveles de estrona y estradiol. Las aromatasas están presentes en el tejido mamario normal y aún en mayor concentración en el tejido maligno ${ }^{(33)}$, lo que explica que la concentración local de estrógenos en post-menopáusicas sea 10 a 20 veces mayor que la sistémica ${ }^{(34)}$.

\section{¿Qué rol juega el consumo del alcohol en el cáncer de mama? \\ Este factor parece ser importante, el RR aumenta de manera dosis dependiente entre las bebedoras, especialmente en post-menopáusicas ${ }^{(35)}$ : \\ - 1.39 (1.16-1.67) por 12 a $18 \mathrm{gr} / \mathrm{d}$ ía \\ - 2.30 (1.51-3.51) por 35 a 45 gr/día}

\section{¿Qué rol juega el uso de anticonceptivos orales en cáncer de mama?}

Los anticonceptivos orales (ACO) (estrógenos + progestina), generan disminución de los ciclos ovulatorios y por ende el efecto estrogénico disminuye. Sin embargo, aumentan el efecto de progesterona con su actividad mitogénica ${ }^{(36)}$. Las características de las diferentes progesteronas es muy variable en los diferentes anticonceptivos, siendo algunas potencialmente más carcinogénicas que otras ${ }^{(37)}$. La medroxiprogesterona (MPA) por ejemplo, incrementa la incidencia de tumores mamarios en monos y perros, pero no en humanos; su verdadero rol permanece en discusión y estudio. Asímismo, se están evaluando las progesteronas naturales como factores de riesgo o protección.

El uso de anticonceptivos orales por tiempo prolongado sólo está asociado a mayor riesgo en mujeres nulíparas con mutación BRCA1 y BRCA2 (38), existiendo otros estudios que incluso niegan ésto. El embarazo produce una mayor diferenciación del tejido mamario, principalmente a nivel lobular, inhibiendo el potencial carcinogénico de los $\mathrm{ACO}^{(39)}$. 


\section{¿Qué son los SERM-s?}

Los moduladores selectivos de receptores estrogénicos, conocidos como SERM-s (entre los que se cuenta el tamoxifeno y el raloxifeno), tienen actividad estrogénica específica, agonista y antagonista según la presencia predominante de receptores $\alpha$ o $\beta$. Vale decir, en algunos tejidos son de un accionar símil a estrógeno y en otros ejercen un rol antagónico. Tienen un papel protector variable sobre el hueso, previniendo la osteoporosis $(2,4 \%$ de aumento en columna y $2 \%$ en cadera, cifra bastante menor que el $7 \%$ que ejercen los estrógenos y los bifosfonatos) ${ }^{(40)}$. Son antagonistas sobre el tejido mamario en presencia de receptores positivos. Reducen el colesterol LDL, pero no generan una mayor protección cardiovascular, por lo cual no se sugiere su uso con ese objetivo. Están asociados a mayor riesgo de trombosis venosa periférica y trombo-embolismo pulmonar, cataratas, hiperplasia y cáncer del endometrio. Los efectos deletéreos descritos son mayores con el uso del tamoxifeno $\mathrm{v} / \mathrm{s}$ raloxifeno ${ }^{(41)}$. Ambos son protectores contra cánceres receptor $\mathrm{E}(+)$ : se ha demostrado que el tamoxifeno disminuye en un $86 \%$ el riesgo de cáncer en pacientes con hiperplasias atípicas en biopsias previas. El raloxifeno es capaz de reducir el riesgo de cáncer invasor en un $65 \%(42,43)$.

\section{¿Qué podríamos decir de la THR y el cáncer de mama?}

Aproximadamente 45 mujeres por 1.000 - sin THR - presentarán cáncer de mama entre los 50 y 70 años (el riesgo aumenta con la edad).

Entre las mujeres con THR que duran de 5 a 15 años, el número de cánceres adicionales será de 2 a 12 por 1.000 mujeres tratadas, según la edad de inicio y duración del tratamiento ${ }^{(18)}$.

Analizando diferentes estudios, el incremento de riesgo de cáncer de mama sólo ha sido demostrado a partir de más de 5 años de uso ininterrumpido (durante este período incluso el placebo representa un riesgo mayor) por lo que su empleo hasta 5 años podría ser recomendado sin mayores riesgos en relación a la mama(18).

El uso exclusivo de estrógenos, en todos los estudios representa un menor riesgo ( $R R=1.2$ (1.021.37)) y se expresa en forma más tardía (sobre 7-15 años), mientras la terapia con estrógeno + progesterona incrementa éste en forma significativa $(R R=1.63$ (1.22-2.18)) y precozmente, desde los 5 años ${ }^{(1,44)}$.

La progesterona se emplea en forma combinada (durante todo el ciclo junto con estrógeno) o secuencial (por tiempo limitado, 12 a 14 días del ciclo). Hasta ahora, los estudios son contradictorios para poder elegir la mejor alternativa que presente el menor riesgo.

El riesgo de cáncer mamario parece aumentar con la duración del tratamiento (incremento anual de
$2,3 \%$ ). Todos los estudios publicados concuerdan que éste desaparece rápidamente (en 1 a 5 años) al suspender el $\operatorname{THR}^{(18,45,46)}$, a valores similares al de mujeres que nunca fueron expuestas y ésto es independiente del tiempo de uso previo(47,9).

Considerando el período de latencia de un cáncer de mama, 6 a 10 años desde su inicio real y el momento de su detección radiológica, el efecto de TRH no debe ser inductor, sino probablemente promotor: aumentaría el crecimiento de pequeños tumores pre-existentes, explicando así los cánceres adicionales que se asocian a su uso. De este modo, se comprende que al suspenderlo el riesgo de cáncer desaparece rápidamente, hasta llegar a su condición inicial.

\section{THR y mortalidad por cáncer mamario}

Según la mayoría de estudios observacionales, el pronóstico de cánceres mamarios manifiestos bajo THR sería más favorable (quizás por el mayor control de las pacientes tratadas) que de los cánceres espontáneos, siendo más diferenciados, de menor tamaño al momento de su diagnóstico y con menor potencial metastásico ${ }^{(18)}$. Otros estudios (WHI 2002 y 2003) señalan lo opuesto(4, 48).

Un efecto desfavorable de THR sobre la incidencia de cáncer mamario debería estar acompañado por el aumento de la mortalidad por esta causa, fenómeno que no fue observado globalmente: algunos estudios demuestran la disminución de mortalidad ${ }^{(49)}$ y otros (MWS) no detectan modificación ${ }^{(46)}$. Sólo el estudio de enfermeras de Boston ${ }^{(45)}$ indica un incremento en mujeres con más de 10 años de THR.

\section{¿Qué rol juegan los fitoestrógenos como alter- nativa a la THR clásica?}

En países del oriente, principalmente en Japón, la incidencia de cáncer mamario es netamente menor que en los países occidentales, atribuyéndose a su rica dieta de fitoestrógenos (FE) principalmente productos de soya (isoflavonoide). Estos FE actúan especialmente en los ER $\beta$, siendo 1.000 a 100.000 veces menos afines que el estradiol por este receptor. Actúan en diferentes niveles: como débiles antiestrógenos, inhiben las enzimas esteroidogénicas aromatasas $(17 \beta-H S D 1$ que convierten la débil estrona en estradiol) e incrementan el SHBG, disminuyendo la fracción libre de estrógenos ${ }^{(50)}$.

El uso de FE por períodos breves ha sido reportado como un factor de proliferación celular pero no generador de cáncer, pudiendo ser un riesgo en mujeres de bajos niveles de estrógeno. Su empleo en la disminución de los fenómenos vasomotores carece de utilidad.

El rol protector en cáncer de mama, ampliamente publicitado, carece de estudios adecuadamente randomizados por problemas metodológicos. Sólo en dosis 
muy alta se podría lograr el efecto benéfico que no se alcanza con el FE de uso habitual. Probablemente la edad de inicio de ingesta, duración de la misma y tipo de FE explican su efecto protector en asiáticas. Por lo anterior, no se lo recomienda como alternativa al uso de estrógenos clásicos de dosificación y de efectos conocidos ${ }^{(51)}$.

\section{¿Se puede proponer TRH a pacientes con riesgo familiar de cáncer de mama?}

Existe evidencia que el número de parientes afectados (de primer grado) aumenta espontáneamente la incidencia de cáncer de mama ${ }^{(52)}$ y se correlaciona con mayor mortalidad, que a su vez aumenta con la edad. ¿Este neto aumento de riesgo contraindica el uso de THR? La respuesta -según diferentes estudios, como el metanálisis de Oxford ${ }^{(16)}$, WHI-brazo estrógenos solo ${ }^{(53)}$ y el estudio publicado en el Lancet (2001)(54) - es no, puesto que el TRH no agrava el riesgo: se demostró que el $\mathrm{RR}$ en mujeres de alto riesgo >50 años es de 1.88 (1.64-2.049) sin THR vs 1.5 (1.1-2.09) con TRH de 5 años de duración.

\section{Estudios epidemiológicos}

Las aseveraciones anteriormente expuestas se basan en estudios epidemiológicos (HERS, WHI, E3N, diferentes metanálisis etc.) con conclusiones a veces discordantes, ya que ninguno de estos estudios puede tomar en cuenta la diversidad de las hormonas utilizadas, su posología y vía de administración. Cabe destacar, que si bien los trabajos recientemente publicados (HERS II, WHI) son prospectivos, randomizados y doble ciego contra placebo, éstos raramente han fijado como objetivo principal la evaluación de riesgo para cáncer mamario. Por lo mismo, tienen limitaciones en la selección de pacientes según edad, nivel socio-económico, factores de riesgo familiar, dietético, alcohol etc. La mayoría de los estudios fueron realizados en EE.UU., con esquema de estrógeno de equino combinado, vía oral (diferente a las mujeres europeas que usan preferentemente la vía transdérmica) y en pacientes de mayor edad (promedio de 64 años/WHI). Sin embargo, por el momento no disponemos de otros trabajos con poder estadístico suficiente.

Los estudios más importantes, evaluando el riesgo del THR son:

a) Estudio de enfermeras de Boston(55): trabajo prospectivo, basado en el seguimiento de 122 mil enfermeras desde 1976. Sus primeros resultados publicados (N Engl J Med 1995) demostraron un aumento de riesgo de $30 \%$ atribuible al uso de THR. Los últimos resultados fueron publicados en $2006^{(45)}$, sobre 28.835 mujeres postmenopáusicas, histerectomizadas, tratadas por estrógenos. Encontraron 934 cánceres, pero el aumento de riesgo sólo apareció después de 20 años de uso de estrógenos conjugados solos.

b) Metanálisis de Oxford (Lancet 1997)(18): analiza el $90 \%$ de los estudios publicados hasta la fecha sobre el efecto de THR, incluyendo 51 publicaciones (31 países) con un total de 53.865 mujeres post-menopausicas (17.949 casos y 35.916 controles), $34 \%$ de las mujeres con $>5$ años de THR. Sus conclusiones son importantes ya que en ellas se basa la información oficial de las guías clínicas sobre el efecto secundario de THR:

- Concluyó que la menopausia disminuye el riesgo de cáncer mamario, independientemente de la edad de su inicio.

- Confirmó un leve aumento del riesgo entre las mujeres con $\mathrm{TRH}(80 \%$ de las mujeres con estrógenos solo): RR global=1.14+0,03 aumentado a 1.35 (1.21-1.49) después de 5 años de uso.

- Precisó la incidencia acumulativa de cáncer mamario: 45 por 1.000 mujeres de entre 50 y 70 años que aumentó (atribuible al TRH) con 2 casos después de 5 años, 6 casos después de 10 años y 12 casos después de 15 años de uso ininterrumpido de estrógenos.

- Según sus conclusiones, este riesgo es comparable con el riesgo asociado a una menopausia tardía: $2.3 \%$ vs $2.8 \%$ por año.

c) HERS I (JAMA 1998)(56): primer estudio prospectivo, randomizado, doble ciego que fue diseñado para evaluar el efecto protector de THR estroprogesterona en mujeres post-menopáusicas con enfermedad coronaria previa. Colateralmente, permitió evaluar algunos riesgos para cáncer mamario.

d) Una campaña mediática se lanzó después de la publicación de Schairer et al. (JAMA 2000) (2) en que analizaron 2.082 canceres en 46.355 mujeres post-menopáusicas (edad promedio de 58 años): $R R=1.2(1.0-1.4)$ para estrógenos solo, y de 1.4 (1.1-1.8) con estro-progesteronas. El incremento de riesgo anual es de 0.01/año (0.002-0.03) y de 0.08/año (0.02-0.16) para los dos grupos respectivamente.

e) El estudio WHI (JAMA 2002)(4) randomizado y doble ciego, reveló un riesgo comparable con la utilización de estrógenos conjugados + MPA: $R R=1.24$ (1.01-1.54), correspondiente en riesgo absoluto a 8 casos adicionales de cáncer mamario para 10.000 año mujer. El aumento de riesgo de cáncer de mama se observó en el grupo de mujeres que han recibido $\mathrm{TRH}$ previo a su inclusión en el estudio. En mujeres jamás tratadas previamente, el aumento no fue significativo; al contrario, en el brazo del estudio, considerando a mujeres histerectomizadas, tratadas con 
estrógenos conjugados solos durante 7.1 años en promedio, se observó una disminución de riesgo ( $R R=0.80$; IC a 95\%: 062-1.04).

El shock cultural se produjo en el verano de 2002, cuando se anunció la suspensión de una rama del estudio WHI. Esto se justificó por el aumento de eventos graves (accidentes vasculares cerebrales e infarto al miocardio en este caso preciso), sobrepasando el límite de seguridad establecido por el comité científico. El número total de cánceres mamarios fue 290, 166 en el grupo TRH y 124 en grupo placebo. Con un hazard ratio (HR) de 1.26 e IC de $95 \%$ (1.00-1.59). El THR causó un aumento absoluto de 8 cánceres (38 vs 30) por 10.000 año mujer.

h) La publicación de la MWS (Millon Women Study) (Lancet 2003)(46), estudio de observación prospectivo, incluyendo 1.084 .110 mujeres de 50 a 64 años (de 1996 a 2001) reforzó la idea de riesgo del TRH: el TRH con estrógeno sólo es responsable de 5 cánceres adicionales por 10.000 año mujer. Los estro-progesteronas aumentan con 19 cánceres por 10.000 AM. Su $\mathrm{RR}$ aumenta con la duración del tratamiento, alcanzando 2.31 (2.08-2.56) después de 10 años.

i) El estudio de WHI 2 (JAMA, 2004)(57), que se refiere al uso de estrógenos en pacientes histerectomizadas, demostró una disminución de cánceres mamarios con 7 casos y un RR de 0.77 (0.59-1.01), es decir no significativo. Confirmó la idea de que el riesgo de estrógenos es menor que el de los estro-progesteronas. En esta misma población no se encontró efecto protector del estrógeno contra el cáncer de colon.

j) Se publicó el estudio francés E3N (Int J Cancer, 2005) $)^{(58)}$, enrolando 98.997 mujeres nacidas entre 1925 y 1950. El RR de cáncer de mama en la población total es de 1.2 (IC 95\% 1.1-1.4). Para estrógeno solo, el riesgo no es significativo: $R R=1.1$ (IC95\%0.8-1.6). Con estrógeno- progesteronas el RR aumenta a 1.3 (1.1-1.5). En este estudio se evidenció diferencia entre las progesteronas sintéticas y naturales: $R R=1.4$ vs 0.9 (0.7-1.2), es decir las formas naturales de progesterona no aumentarían el riesgo. Esto generó la idea de iniciar el THR en el periodo peri-menopáusico con estrógenos y progesteronas naturales. Sin embargo, se requiere de otros estudios prospectivos, randomizados, diseñados especialmente para su confirmación.

Considerando estos resultados ¿como podemos informar a nuestras pacientes?

No es discutible el rol favorable que ejercen los estrógenos frente a las otras alternativas en presen- cia de severos signos vasomotores o en relación a síntomas periféricos de déficit hormonal:

- El TRH está indicado para tratar el síndrome de climaterio en la dosis más baja posible y durante el tiempo más corto en la peri-menopausia(59-61). Se recomienda una reevaluación anual, enfatizando en los factores de riesgo personales según el modelo de Gail.

- $\quad$ El uso de estrógeno solo (en histerectomizadas) por menos de 7 años no representa factor de riesgo. La asociación preferente de una progesterona natural parece ser menos riesgosa y más recomendable al usar terapia de reemplazo en presencia de útero.

- $\quad$ La utilización de la vía transdermica(62) y nasal vs oral evita parte de los efectos deletéreos atribuibles a los estrógenos orales (como son el tromboembolismo, litiasis vesicular), pero carece de beneficio en el perfil lipídico, lo que sí tienen los orales.

- Recordar que los antecedentes personales de cáncer mamario representan una contraindicación absoluta de THR.

- $\quad$ El THR en la menopausia aumenta el riesgo de cáncer mamario, pero es importante explicar en valores absolutos el numero de cánceres adicionales, atribuibles al uso de THR (8 en WHI, 2 a 12 en MWS, según el tipo y duración del tratamiento), mientras 1250 y 833 mujeres respectivamente (WHI y MWS) no tendrán cáncer este año.

- $\quad$ Se debe también considerar en el balance los beneficios que significa THR para el riesgo de cáncer de colon.

- La utilización de fitoestrógenos como alternativa a la THR no aparece avalada por publicaciones, de tal manera que no parece una alternativa válida hasta el momento.

- $\quad$ El empleo de estrógenos con otros fines a los antes mencionados no está recomendado por la literatura.

- $\quad$ El TRH no es el único tratamiento de los síntomas y complicaciones de la menopausia; a las pacientes se debe informar sobre las alternativas disponibles. Si bien contamos con excelentes estudios prospectivos randomizados para precisar los beneficios de SERM y de los bifosfonatos que demuestran su eficacia, inconvenientes (ej. bochornos, epigastralgias) y contraindicaciones (ej. patología endometrial pre-existente, reflujo) también deben ser detallados.

Con la menopausia se genera una disminución de la masa ósea, la cual debe ser preservada con ejercicios, consumo de productos lácteos, disminución del consumo de café y carne roja. Los estrógenos protegen la masa ósea, pero no están 
indicados como tratamiento de primera línea en la prevención de la osteoporosis.

\section{Conclusiones}

Con respecto a la pregunta: ¿El THR aumenta el riesgo del cáncer de mama? no existe aún respuesta definitiva. Vemos que los efectos son diferentes según la hormona utilizada, posología, vía de administración, esquemas de tratamiento, duración y factores de riesgo personal.

Por el momento, podríamos resumirlo de la siguiente manera:

- Globalmente, un THR parece inducir el mismo efecto que una menopausia tardía, es decir disminuye el rol protector de la menopausia respecto al riesgo del cáncer mamario.

- Los estrógenos solos no aumentan el riesgo o éste es mínimo, expresándose con su uso prolongado (sobre 5 a 6 años).

- No obstante, en mujeres menopáusicas con útero, donde es necesario el empleo de progesterona, ésta claramente aumenta el riesgo. Aún permanece en estudio que las progesteronas naturales no modificarían el riesgo.

- La utilización de la vía transdérmica no aumenta el riesgo tromboembólico pero aún no hay estudios confiables respecto al riesgo asociado a cáncer de mama.

- El aumento de cáncer mamario atribuible a algunas formas del THR, parece ser más bien un efecto promotor y en ningún caso inductor.

La decisión final de THR debe ser compartida con la paciente, después de entregarle una información objetiva, clara y apropiada durante la consulta, basada en evidencias validadas y tomando en cuenta el análisis personalizado de su riesgo.

Cuando vemos la complejidad de la asociación THR-cáncer, con sus múltiples variables e incertidumbres, es comprensible que la toma de decisión compartida frecuentemente conlleve a una actitud "evitativa" por parte del médico, en nombre de "la precaución". Este hecho es lamentable, ya que queda en la ambigüedad la responsabilidad del médico de sugerir una conducta.

\section{Bibliografía}

1. Magnusson C, Baron JA, Correia N, Bergstrom R, Adami HO, person I. Breast câncer risk following long term oestrogen-progestin replacment therapy. Int $\mathrm{J}$ Cancer 1999; 81: 339-344.

2. Schairer C, Lubin J, Trisi R, Sturgeon S, Brinton L, Hoover R. menopausal estrogen and estrogen-progestin replacement therapy and breast cancer risk. JAMA 2000; 283: 485-491.

3. Ross RK, Paganini-Hill A, Wan PC, Pike MC. Effect of hormone replacements therapy on breast cancer risk: estrogen versus estrogen plus progestin. J Natl
Cancer Inst 2000; 92(4): 328-32.

4. WHI. Writing group for the women's health initiative investigators. Risks and benefits of estrogen plus progestin in healthy post menopausal women. JAMA 2002; 288: 321-333.

5. Hersh AL, Stefanick ML, Stafford RS. National use of post-menopausal hormone therapy: annual trends and response to recent evidence. JAMA 2004 7; 291(1): 47-53.

6. Espie M, Gorins A: Epidemiologie du cancer du sein. Le Sein, 1 vol, Edition Eska, París, 1995; 339-347.

7. Consensus on menopause of the European Menopause Society. Human Reprod. 1996; 11: 976-979.

8. Beatson GT. On the treatment of inoperable cases of carcinoma of the mamma: suggestions for a new method of treatment, with illustrative cases. Lancet 1896; 2: 104-107.

9. Mark Clemons and Paul Goss. Estrogen and the risk of breast cancer. N Engl J Med 2001; 344: 276-285.

10. Pike MC, Spicer DV, Dahmoush L, Press MF. Estrogens, progestogens, normal breast cell proliferation and breast cancer risk. Epidemiol Rev 1993, 15: 17-35.

11. Applanat MP, Buteau-Lozano $\mathrm{H}$, Herve MA, Corpet A. Vascular endothelial growth factor is a target gene for estrogen receptor and contributes to breast cancer progression. Adv Exp Med Biol. 2008; 617: 437-44.

12. Khan SA, Rogers MA, Obando JA, Tamsen A. Estrogen receptor expression of benign breast epithelium and its association with breast cancer. Cancer Res 1994; 54: 993-997.

13. Khan SA, Rogers MA, Obando JA, Tamsen A. Estrogen receptor expression of benign breast epithelium and its association with breast cancer. Cancer Res 1994; 54: 993-997.

14. Seema A. Khan, Mary A. M. Rogers, Juan A. Obando and Ali Tamsen Estrogen Receptor Expression of Benign Breast Epithelium and Its Association with Breast Cancer Cancer Research 1994; 54: 993-997.

15. Hsieh C, Trichopoulos D, Katsouyanni K, et al. Age at menarche, age at menopause, height and obesity as risk factors for breast cancer: associations and interactions in an international case-control study. Int J Cancer 1990; 46: 796.

16. Layde PM, Webster LA, Baughman AL, et al. The independent associations of parity, age at first full term pregnancy, and duration of breastfeeding with the risk of breast cancer. Cancer and Steroid Hormone Study Group. J Clin Epidemiol 1989; 42: 963-973.

17. Jensen $A$, Sharif $H$, Olsen JH, Kjaer SK. Risk of breast cancer and gynecologic cancers in a large population of nearly 50,000 infertile Danish women. Am J Epidemiol 2008; 168(1): 49-57.

18. Collaborative group on hormonal factors in breast cancer. Breast cancer and hormone replacement therapy: collaborative reanalysis of data from 51 epidemiological studies of 52705 women with breast cancer and 108.411 women without breast cancer. Lancet 1997: 350: 1047-1059.

19. National Institute on Aging U. S. Department of Health and Human Services Public Health Service. National Institutes of Health July 2006.

20. Albrektsen G, Heuch I, Kvale G. Joint effects on cancer risk of age at childbirth, time since birth and attained age: circumventing the problem of collinearity. Stat 
Med 1999; 18: 1261-1277.

21. Victor G. Vogel, Emanuela Taioli. Have We Found the Ultimate Risk Factor for Breast Cancer? J Clin Oncol 2006; 24: 1791-1794.

22. Fisher B; Dignam J; Wolmark N; Wickerham DL; Fisher ER; Mamounas E; Smith R; Begovic M; Dimitrov NV; Margolese RG; Kardinal CG; Kavanah MT; Fehrenbacher L; Oishi RH. Tamoxifen in treatment of intraductal breast cancer: National Surgical Adjuvant Breast and Bowel Project B-24 randomised controlled trial. Lancet 1999; 12(353): 1993-2000.

23. Richard J. Santen, Jo Ann Pinkerton, Christopher McCartney and Gina R. Petroni: Risk of Breast Cancer with Progestins in Combination with Estrogen as Hormone Replacement Therapy J Clin Endocrinol Metab 2001; 86: 16-23.

24. Bruzzi P, Negri E, La Vecchia C, et al. Short term increase in risk of breast cancer after full term pregnancy. BMJ 1988; 297: 109-125.

25. Layde PM, Webster LA, Baughman AL, Wingo PA, Rubin GL, Ory HW. Cancer and Steroid Hormone Study Group. The independent associations of parity, age at first full term pregnancy, and duration of breastfeeding with the risk of breast cancer. J Clin Epidemiol 1989; 42(10): 963-73.

26. Henderson BE, Ross RK, Judd HL, Krailo MD, Pike $\mathrm{MC}$. Do regular ovulatory cycles increase breast cancer risk? Cancer 1985; 56: 1206-1208.

27. Kaaks R, Berrino F, Key T, et al. Serum sex steroids in premenopausal women and breast cancer risk within the European Prospective Investigation into Cancer and Nutrition (EPIC). J Natl Cancer Inst 2005; 97: 755 768.

28. Clemons M, Goss P. Estrogen and the risk of breast cancer. N Engl J Med 2001 25; 344(4): 276-285.

29. Lupulescu A. Estrogen use and cancer incidence: a review. Cancer Invest 1995; 13: 287-295.

30. Key T, Appleby P, Barnes I, Reeves G. Endogenous sex hormones and breast cancer in post-menopausal women: reanalysis of nine prospective studies. J Natl Cancer Inst 2002; 94: 606-616.

31. Van D, Brandt PA, Spiegelman D, Yaun SS, et al. Pooled analysis of prospective cohort studies on height, weight, and breast cancer risk. Am J Epidemiol 2000; 152: 514

32. James D, Yagerer, Nancy E. Davidson ,M.D Estrogen Carcinogenesis in Breast Cancer N Engl J 2006 354: 270-282.

33. Jefcoate CR, Liehr JG, Santen RJ, et al. Tissue-specific synthesis and oxidative metabolism of estrogens. In estrogens as endogenous carcinogens in the breast and prostate. Journal of the National Cancer Institute mgraph N27. Bethesda, Md:National Cancer Institute, 2000: 95-112.

34. Pasqualini JR, Chetrite G, Blacker C, Feinstein MC, Delalonde L, Talbi M, Maloche C. Concentrations of estrone, estradiol, and estrone sulfate and evaluation of sulfatase and aromatase activities in pre- and postmenopausal breast cancer patients. Journal of Clinical Endocrinology and Metabolism 1996; 81: 1460-1464.

35. Longneker MP, Newcomb PA, Mittendorf R. et coll. Risk of breast cancer in relation to lifetime alcohol consumption. J Nat Cancer Inst 1995; 87: 923-929.

36. Pike MC, Henderson BE, Krailo MD, Duke A, Roy S.
Breast cancer in young women and use of oral contraceptives: possible modifying effect of formulation and age at use. Lancet 1983; 2: 926-930.

37. James R Cerhan. Oral contraceptive use and Breast cancer risk: Current Status Mayo Clin Proc Oct 2006; 81(10): 1287-1289.

38. Ursin G, Henderson BE, Haile RW, et al. Does oral contraceptive use increase the risk of breast cancer in women with BRCA1/BRCA2 mutations more than in other women? Cancer Res 1997; 57: 3678-3681.

39. James R Cerhan. Oral contraceptive use and Breast cancer risk: Current Status Mayo Clin Proc Oct 2006; 81(10): 1287-1289.

40. Once-weekly alendronate $70 \mathrm{mg}$ and raloxifene $60 \mathrm{mg}$ daily in the treatment of postmenopausal osteoporosis. Luckey M; Kagan R; Greenspan S; Bone H; Kiel RD; Simon J; Sackarowitz J; Palmisano J; Chen E; Petruschke RA; de Papp AE Menopause 2004; 11(4): 405-1558.

41. Cummings SR, Duong T, Kenyon E, Cauley JA, Whitehead M, Krueger KA, for the multiple outcomes of raloxifene evaluation (more) trial. Serum estradiol level and risk of breast cancer during treatment with raloxifen. JAMA 2002; 287: 216-220.

42. Rowan T, Chlebowski. Reducing the Risk of Breast Cancer N Engl J Med 2002; 343: 191-198.

43. SR. Cummings, S Eckert KA. Krueger; D Grady; Trevor J. Powles; JA. Cauley; L Norton; T Nickelsen; NH. Bjarnason; M Morrow; ME. Lippman; D Black; J E. Glusman; A Costa V. C Jordan The Effect of Raloxifene on Risk of Breast Cancer in Postmenopausal Women Results From the MORE Randomized Trial JAMA 1999; 281: 2189-2197.

44. Shah NR, Borenstein J, Dubois RW. Postmenopausal hormone therapy and breast cancer: a systematic review and meta-analysis. Menopause 2005; 12: 668-678.

45. Chen WY, Manson JE, Hankinson SE. et coll. Unopposed estrogen therapy and the risk of invasive breast cancer. Arch Intern Med 2006; 166: 1027-1032.

46. Million women study collaborators. Breast cancer and hormone-replacement therapy in the Million Women Study. Lancet 2003; 362: 419-427.

47. Post-menopausal oestrogen therapy. Lyon, France: International Agency for Research on Cancer, 1999.

48. Chlebowsky RT, Hendrix SL, Stefanick ML, et coll. For the whi investigators. Influence of estrogen plus progestin on Breast cancer and mammography in healthy postmenopausal women. The Women's Health Initiative Randomized Trial. JAMA 2003; 289: 3243-3253.

49. Fletcher AS, Erbas B, Kavanagh AM, Hart S, Rodger A, Gertig DM. Use of hormone replacement therapy (HRT) and survival following breast cancer diagnosis. The Breast 2005; 14: 192-200.

50. Suman Rice and Saffron A Whitehead Phytoestrogens and breast cancer-promoters or protectors? EndocrineRelated Cancer 2006; 13 (4): 995-1015.

51. R. M. Harris, D. M. Wood, L. Bottomley, S. Blagg, K. Owen, P. J. Hughes, R. H. Waring and C. J. Kirk Phytoestrogens Are Potent Inhibitors of Estrogen Sulfation: Implications for Breast Cancer Risk and Treatment $J$ Clinic Endocrinol Metab 2004; 89(4): 1779-1787.

52. Rippy I, Marsden J, Is HRT justified for symptom management in women at hight risk of developing breast cancer? Climateric 2006; 9: 404-415. 
53. Stefanick ML, Anderson GL, Margolis KL. et coll. Effects of conjugated equine estrogens on breast cancer and mammography screening in postmenopausal women with hysterectomy JAMA 2006; 295: 1647-1657.

54. Collaborative group on hormonal factors in breast cancer. Familial breast cancer: collaborative reanalysis of individual data from 52 epidemiological studies, including 58209 women with breast cancer and 101986 women without disease. Lancet 2001; 358: 1389-1399.

55. Grodstein F, Stampfer MJ, Manson JE, et al. Postmenopausal estrogen and progestin use and the risk of cardiovascular disease. N Engl J Med 1996; 335: 453-461.

56. S Hulley D, Grady T, Bush C, Furberg D, Herrington B Riggs, E Vittinghoff. for the Heart and Estrogen/ progestin Replacement Study (HERS) Research Group Randomized Trial of Estrogen Plus Progestin for Secondary Prevention of Coronary Heart Disease in Postmenopausal Women JAMA. 1998; 280: 605-613.
57. The women's health initiative streering comité: Effects of conjugated equine estrogen in postmenopausal women with hysterectomy: the women's health initiative randomized controlled trial. JAMA 2004; 291: 1701-1712.

58. Fournier A, berrino F, Riboli E, Avenel V, Clavel ChapeIon $\mathrm{F}$. Breast cancer risk in relation to different types of hormone replacement therapy in the E3N-EPIC cohort. Int J Cancer 2005; 114: 448-454.

59. Karin H. Humphries and Sabrina Gill.Risk and Benefits of Hormone replacement Therapy: The evidence speaks. CAMJ 2003, 168(8).

60. SD. Redd, K.M. Newton, Andrea Z. La Croix Indications for hormone therapy the post-womens Health Initiative era. Endocrinology and Metabolism Clinics of North American 2004; 33: 691-715.

61. Freeman R, Lewis RM. The Therapeutic Role of Estrogens in Postmenopausal Women Endocrinology and Metabolism Clinics of North America 2004; 33(4): 771-789

62. Tanping Wong, Nirav R Shah. Breast cancer from oral and transdermal estradiol: a cohort study of Finnish women. Women's Health 2007; 3(3): 321-324. 